\title{
WIRELESS POWERED COMMUNICATION NETWORKS WITH IMPERFECT CHANNEL STATE INFORMATION AND NON-IDEAL CIRCIUT POWER CONSUMPTION
}

\author{
Slavče Pejoski, Zoran Hadži-Velkov \\ Faculty of Electrical Engineering and Information Technologies, \\ "Ss. Cyril and Methodius" University in Skopje, \\ Rugjer Bošković bb, P.O. box 574, 1001 Skopje, Republic of Macedonia \\ \{slavchep, zoranhv\}@feit.ukim.edu.mk
}

\begin{abstract}
A b s t r a c t: Assuming imperfect channel state information (CSI), we propose a resource allocation policy that maximizes the achievable sum rate in uplink and accounts for the power consumption due to non-ideal circuitry. The proposed policy assumes that the energy harvesting units (EHUs) in a single transmission spend only a part of the available harvested energy in their batteries, and save the rest of it for future use. By simulation, we evaluate the influence of the number and the power of the pilot symbols, and the number of EHUs to the achievable sum rate. Additionally, we estimate the loss in the achievable sum rate compared to the system with perfect CSI.
\end{abstract}

Key words: energy harvesting; uplink TDMA WPCN; channel estimation; imperfect CSI; non-ideal cirquit power

\section{БЕЗЖИЧНО НАПОЈУВАНИ КОМУНИКАЦИСКИ МРЕЖИ СО НЕСОВРШЕНА ИНФОРМАЦИЈА ЗА СОСТОЈБАТА НА КАНАЛОТ И ПОТРОШУВАЧКАТА НА МОК์НОСТ ПОРАДИ НЕИДЕАЛНИ ЕЛЕКТРИЧНИ КОЛА}

\begin{abstract}
А п с т р а к т: Во трудот предлагаме механизам за доделување ресурси со кој се максимира збирната информациска брзина во една безжично напојувана телекомуникациска мрежа со неидеални електрични кола и неидеална информација за каналот. Предложената шема претпоставува дека јазлите кои вршат „жетва на енергија“ при секое праќање информација трошат само дел од расположливата енергија во нивните батерии, а остатокот го чуваат за следни праќања. Перформансите на еден ваков систем се анализирани преку компјутерски симулации, при што е определено влијанието на бројот и моќноста на пилотските симболи и на бројот на јазли врз збирната информациска брзина. Дополнително е извршена споредба со систем со совршена информација за состојбата на каналот.
\end{abstract}

Клучни зборови: жетва на енергија; пренос на информација кон базна станица во безжично напојувана телекомуникациска мрежа; процена на каналот; несовршена информација за состојбата на каналот; потрошувачка на моќност заради неидеални електрични кола

\section{INTRODUCTION}

The wireless powered communication networks (WPCNs) have recently emerged as a highly promising solution for self-sufficient communication in energy-constrained environments (c.f. [1] and references therein). Most of the studies on WPCNs typically optimize the uplink of a centralized network, which consists of a base station (BS) and multiple energy harvesting (EH) units (EHUs), with resource sharing using time division multiple access (TDMA), assuming perfect channel state information (CSI) at both the transmitter and the receiver, $[1-5]$. In practice, each node in the net- 
work carries out channel estimation, which makes the available CSI imperfect. The impact of imperfect CSI over the conventional communication systems has been well understood [6-10]. In the WPCN context, however, this issue has not been tackled, except in [11]. The paper [11] proposes power allocation policy when the BS transmits energy and information to the EHUs over the downlink. The study of the influence of imperfect CSI is more challenging when the EHUs transmit information over the uplink due to the uncertainty in the available energy at the EHUs (consequence of the energy harvesting process). Additionally, due to mathematical intractability, only a few studies take into account the non-ideal circuit power consumption of the EH nodes, which is very important to asses the performance of practical WPCNs [3, 4]. Here we assume that the EHUs are equipped with battery that can store the harvested energy and using the results from [12] for the battery model and the results from [10] for imperfect CSI at the transmitter we estimate the achievable sum rate over the uplink of WPCNs taking into account for the power consumption due to the nonideal circuitry, and determine the optimal power allocation policy that maximize the achievable sum rate. Using simulations we investigate the influence of the key parameters (the number and the power of the pilot symbols and the number of EHUs) on the achievable sum rate.

The first contribution of our work comes from formulating the problem of uplink sum rate maximization in a TDMA based WPCN that takes into account the imperfect CSI, due to channel estimation, the uncertainty of the available energy at the EHUs and the power consumption due to nonideal circuitry. The second contribution comes from finding the resulting solution which leads to asymptotically optimal policies for allocation of BS transmit power, EHUs' transmit power, and time sharing among the BS and the EHUs. The last contribution comes from estimating the influence of the key system parameters on the achievable sum rate.

\section{SYSTEM MODEL}

We consider a TDMA-based WPCN, which consists of a half-duplex BS and $K$ half-duplex EHUs, each equipped with a single antenna. The fading in the channel between the BS and each EHU is a stationary and ergodic random process, and follows the block fading model, i.e. the chan- nel is constant during each block (epoch) but changes randomly from one block to the next. The duration of each epoch, coinciding with a single TDMA frame, is assumed equal to $T$, containing $N$ symbols. The number of epochs $M$ is assumed to satisfy $M \rightarrow \infty$. Each TDMA frame consists of the following consecutive phases (Figure 1): a training phase (uplink), an EH phase (downlink), and $K$ non-overlapping information transmission (IT) phases (uplink). The training phase helps the BS to acquire an (imperfect) estimate of the channels between the BS and all the EHUs. It has a fixed duration and consists of $K$ subintervals, with each subinterval carrying the training sequence from an EHU, which consists of $N_{p}$ symbols of duration $\tau_{p} T$. Each EHU transmits its training sequence at power $P_{p}$. Similar to [12, Example 2], this power is available at the EHUs in almost each epoch (c.f. Section 2.3). In epoch $i$, the BS broadcasts energy at power $P_{0}(i)$ during the EH phase of duration $\tau_{0}(i) T$, and the $k$-th EHU transmits information at power $P_{k}(i)$ during its respective IT phase of duration $\tau_{k}(i) T$. Time sharing parameters $\tau_{p}, \tau_{0}(i)$, and $\tau_{k}(i)$ satisfy
\[ K \tau_{p}+\tau_{0}(i)+\sum_{k=1}^{K} \tau_{k}(i)=1, \quad \forall i \]

The BS transmit power is limited by a maximum power, $P_{\max }$ (i.e. $0 \leq P_{0}(i) \leq P_{\max }$ ), and an average power, $P_{a v g}$ (i.e. $\left.\mathrm{E}\left[P_{0}(i) \tau_{0}(i)\right] \leq P_{a v g}\right)$.

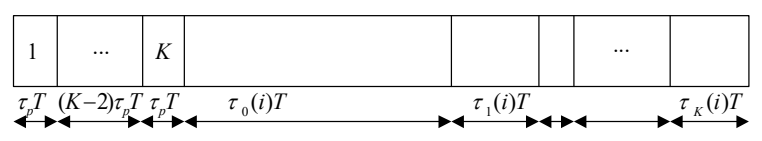

Fig. 1. Time organization of a single epoch

\subsection{Channel estimation}

During the training phase of each TDMA frame, the BS estimates its channel to each of the EHUs. Let us denote the estimated complex channel coefficient of the BS-EHU $k$ channel by $\hat{F}_{k}(i)$, whereas its actual value by $F_{k}(i)$. The channel estimation is realized using minimum mean square error (MMSE) estimator [6] (the MMSE estimator guarantees that the channel estimation error is uncorrelated to the estimated channel gain which is needed to obtain the achievable rate), and the 
channel estimation error is given by $\tilde{F}_{k}(i)=F_{k}(i)-\hat{F}_{k}(i)$. For convenience, the corresponding channel power gains are normalized as $\hat{x}_{k}(i)=\left|\hat{F}_{k}(i)\right|^{2} / N_{0}$ and $x_{k}(i)=\left|F_{k}(i)\right|^{2} / N_{0}$, respectively, where $N_{0}$ is the additive white Gaussian noise (AWGN) power, and $x_{k}(i)$ has average value $E\left[x_{k}(i)\right]=\Omega_{k}$. The normalized variance of the channel estimation error is denoted by $\sigma_{k}^{2}=\mathrm{E}\left[\left|\tilde{F}_{k}(i)\right|^{2}\right] / N_{0}$.

Assuming $F_{k}(i)$ are i.i.d. complex Gaussian random variables, $\sigma_{k}^{2}$ is given by normalizing the expression in [11], above Eq. (1):

$$
\sigma_{k}^{2}=\frac{\mathrm{E}\left[x_{k}(i)\right]}{1+P_{p} \mathrm{E}\left[x_{k}(i)\right] N_{p}}=\frac{\Omega_{k}}{1+P_{p} \Omega_{k} N_{p}} .
$$

Thus, $P_{p}$ and $N_{p}$ are parameters that control the error variance. Increase in their values leads to better channel estimation, but reduces the resources available for the EH and IT phases.

\subsection{EHUs' battery model and non-ideal power consumption}

The EHUs are equipped with rechargeable $\mathrm{EH}$ batteries with unlimited storage capacity, in which they store the harvested RF energy from the BS. Let $B_{k}(i-1)$ denote the available energy in the battery of EHU $k(1 \leq k \leq K)$ at the beginning of epoch $i$. The amount of harvested energy by $k$-th EHU during the EH phase of epoch $i$ is:

$$
E_{h, k}(i)=\eta_{k} x_{k}(i) N_{0} P_{0}(i) \tau_{0}(i) T,
$$

where $\eta_{k}$ is the EH conversion efficiency of this EHU, $0<\eta_{k} \leq 1$. It should be noticed that (2) contains the true power channel gain $x_{k}(i)$, not the channel estimate $\hat{x}_{k}(i)$, because (2) determines the actual harvested power.

In practical EH transmitters, besides the transmit power, an additional power, $p_{c}$, is also consumed due to non-ideal circuit power consumption [3]. We assume that the $k$-th EHU consumes energy only when transmitting (in both: the training phase and the IT phase), and that the power $p_{c}$

is constant independent of the EHU's transmit power. Thus, the total amount of stored energy at the end of the EH phase of epoch $i$ is $B_{k}(i-1)+E_{h, k}(i)-\left(P_{p}+p_{c}\right) \tau_{p} T$ where we have assumed that $B_{k}(i-1)$ is almost always greater than
$\left(P_{p}+p_{c}\right) \tau_{p} T$ (this assumption will be justified later in this section).

During its IT phase, the $k$-th EHU can spend a part of the total amount of the energy stored in its battery. Let $P_{d, k}(i)$ denote the desired transmit power of the $k$-th EHU in epoch $i$. However, this amount of power may not necessarily be available in the battery. Therefore, the actual transmit power of the $k$-th EHU can be written as

$$
P_{k}(i)=\left(\min \left\{\frac{B_{k}(i-1)+E_{h, k}(i)-\left(P_{p}+p_{c}\right) \tau_{p} T}{\tau_{k}(i) T}-p_{c}, P_{d, k}(i)\right\}\right)
$$

where $(x)^{+}=\max \{0, x\}$. Thus, the energy of the battery of $k$ th EHU at the end of the $i$-th epoch is:

$$
B_{k}(i)=B_{k}(i-1)+E_{h, k}(i)-\left(P_{k}(i)+p_{c}\right) \tau_{k}(i) T-\left(P_{p}+p_{c}\right) \tau p^{T} .
$$

According to [12, Theorem 4], in the asymptotic case of $M \rightarrow \infty$, (3) reduces to $P_{k}(i)=P_{d, k}(i)$ when the average consumed power by the EHU is less than or equal to the average harvested power, i.e.

$$
\left(P_{p}+p_{c}\right) \tau_{p}+\mathrm{E}\left[\left(P_{d, k}(i)+p_{c}\right) \tau_{k}\right] \leq \mathrm{E}\left[E_{h, k}(i) / T\right],
$$

Based upon the set of (imperfectly) estimated channels, in each epoch we define the vector $\hat{\mathbf{x}}=\left[\hat{x}_{1}(i), \ldots, \hat{x}_{K}(i)\right]$. Due to the assumed channel ergodicity, it is possible to drop the index $i$ and present the proposed resource allocation policy in terms of the channel fading state $\hat{\mathbf{x}}$.

\subsection{Achievable rate}

In this paper we aim at obtaining optimal power allocations $P_{0}(\hat{\mathbf{x}})$ and $P_{d, k}(\hat{\mathbf{x}})$, and timesharing allocations $\tau_{0}(\hat{\mathbf{x}})$ and $\tau_{k}(\hat{\mathbf{x}})$, which maximize the sum rate over the uplink (to simplify the notation, we leave out $\hat{\mathbf{x}}$ ).

Assuming power allocation policy $P_{d, k}$ and time sharing policy $\tau_{k}$, the achievable rate of EHU $k$ is:

$$
\mathrm{E}\left[\tau_{k} \log _{2}\left(1+\frac{\hat{x}_{k} P_{d, k}}{1+\sigma_{k}^{2} P_{d, k}}\right)\right]
$$


The rate given by (6) refers to the capacity lower bound of a point to point link with imperfect CSI at both transmitter and receiver [6-10], which also applies to our system model since each EHU essentiality transmits over an orthogonal channel. When perfect CSI is not available, and the channel is in a single realization of the state $\hat{\mathbf{x}}$, the rate $\tau_{k} \log _{2}\left(1+\frac{\hat{x}_{k} P_{d, k}}{1+\sigma_{k}^{2} P_{d, k}}\right)$ cannot be achieved, but the average rate (6) can be achieved by transmitting at fixed rate but with variable (state-specific) transmit power. This is referred to as the single codebook with dynamic power allocation scheme [13].

\subsection{Resource allocation policy}

Based upon (6), we now determine the user scheduling, power allocation at the BS and EHUs, and the EH/IT time sharing that maximize the sum rate over the uplink according to the following optimization problem:

$$
\begin{aligned}
& \underset{\tau_{k}, \tau_{0}, P_{0}, P_{d, k}}{\operatorname{Maximize}} \sum_{k=1}^{K} \mathrm{E}\left[\tau_{k} \log \left(1+\frac{\hat{x}_{k} P_{d, k}}{1+\sigma_{k}^{2} P_{d, k}}\right)\right] \\
& \text { s.t. } \\
& C 1:\left(P_{p}+p_{c}\right) \tau_{p}+\mathrm{E}\left[\left(P_{d, k}+p_{c}\right) \tau_{k}\right] \leq \mathrm{E}\left[N_{0} \eta_{k} z_{k} P_{0} \tau_{0}\right] \forall k \\
& C 2: \mathrm{E}\left[P_{0} \tau_{0}\right] \leq P_{a v g} \\
& C 3: 0 \leq P_{0} \tau_{0} \leq P_{\max } \tau_{0}, \forall \hat{\mathbf{x}} \\
& C 4: \sum_{k=1}^{K} \tau_{k}=\tau_{g}-\tau_{0}, \forall \hat{\mathbf{x}} \\
& C 5: 0 \leq \tau_{k} \leq \tau_{g}, \forall \hat{\mathbf{x}}, \forall k \\
& C 6: 0 \leq \tau_{0} \leq \tau_{g}, \forall \hat{\mathbf{x}} \\
& C 7: 0 \leq P_{d, k} \tau_{k}, \forall \hat{\mathbf{x}}, \forall k
\end{aligned}
$$

where $\tau_{g}=1-K \tau_{p}$. The constraint $C 1$ is obtained using (5) and calculating the expectation over $x_{k}$ given $\hat{\mathbf{x}}$ in the right side of (5) which leads to the substitute $z_{k}=\mathrm{E}\left[x_{k} \mid \hat{\mathbf{x}}\right]$. For the special case when $F_{k}(i)$ are i.i.d. complex Gaussian random variables we have $z_{k}=\hat{x}_{k}+\sigma_{k}^{2}$. In the general case, when $\mathrm{E}\left[x_{k} \mid \hat{\mathbf{x}}\right]$ is hard to calculate and $\sigma_{k}^{2}=\mathrm{E}\left[\hat{x}_{k}\right]$ , we can use $z_{k}=\hat{x}_{k}<\mathrm{E}\left[x_{k} \mid \hat{\mathbf{x}}\right]$.

The solution of Problem (7) is given by the following theorem:

Theorem 1: Optimally, a given TDMA frame should contain either an EH phase or an IT phase of duration $\tau_{g}$ : an EH phase when $\sum_{k=1}^{K} N_{0} \eta_{k} z_{k} \mu_{k}>\lambda$, and an IT phase of a single selected EHU when $\sum_{k=1}^{K} N_{0} \eta_{k} z_{k} \mu_{k}<\lambda$. Thus, the BS transmit power is selected as

$$
P_{0}^{*}= \begin{cases}P_{\max }, & \sum_{k=1}^{K} N_{0} \eta_{k} z_{k} \mu_{k}>\lambda \\ 0, & \text { otherwise }\end{cases}
$$

with duration $\tau_{0}^{*}=\tau_{g}$, where $z_{k}$ is equal to $\mathrm{E}\left[x_{k} \mid \hat{\mathbf{x}}\right]$ In the IT phase, the optimal power of the $k$-th EHU is:

$$
P_{d, k}^{*}=\frac{\left(1+\frac{2 \sigma_{k}^{2}}{\hat{x}_{k}}\right)\left(\sqrt{1+\frac{4 \sigma_{k}^{2}\left(\hat{x}_{k}+\sigma_{k}^{2}\right)}{\left(\hat{x}_{k}+2 \sigma_{k}^{2}\right)^{2}}\left(\frac{\hat{x}_{k}}{\mu_{k}}-1\right)^{+}}-1\right)}{2 \sigma_{k}^{2}\left(1+\sigma_{k}^{2} / \hat{x}_{k}\right)}
$$

and, the scheduled EHU is determined by

$$
s=\arg \max _{k=1, \ldots, K} \log \left(\frac{\hat{x}_{k} / \mu_{k}}{\left(1+\sigma_{k}^{2} P_{d, k}^{*}\right)^{2}}\right)-P_{d, k}^{*} \mu_{k}-p_{c} \mu_{k} .
$$

The constants $\lambda$ and $\mu_{k}$ are determined from $C 2$ and $C 1$, respectively, when equality is applied.

Proof: Please refer to Appendix A. Note that (9) corresponds to [8, Eq. (3)].

\subsection{Power and time-sharing allocation: Perfect CSI case}

Following the same approach from Appendix $\mathrm{A}$, it is easy to obtain the optimal solutions when $\sigma_{k}^{2}=0, \forall k$. Namely, when perfect CSI is available at both transmitter and receiver, optimally, a given TDMA frame should contain either an EH phase or an IT phase of duration $\tau_{g}$ : an EH phase when $\sum_{k=1}^{K} N_{0} \eta_{k} x_{k} \mu_{k}>\lambda$, and an IT phase of a single selected EHU when $\sum_{k=1}^{K} N_{0} \eta_{k} x_{k} \mu_{k}<\lambda$. In the EH phase, the transmit power is chosen as:

$$
P_{0}^{*}= \begin{cases}P_{\max }, & \sum_{k=1}^{K} N_{0} \eta_{k} x_{k} \mu_{k}>\lambda \\ 0, & \text { otherwise }\end{cases}
$$


for $\tau_{0}^{*}=\tau_{g}$, and for the IT phase the transmit power of the EHU is chosen as:

$$
P_{d, k}^{*}=\left(\frac{1}{\mu_{k}}-\frac{1}{x_{k}}\right)^{+}
$$

with optimal selection policy, where the index of the selected EHU is determined by

$$
s=\arg \max _{k=1, \ldots, K} x_{k} / \mu_{k} .
$$

Eqs. (12) and (13) correspond to the well known optimal power allocation and user scheduling policy that maximize the sum rate over the uplink of a conventional single-cell wireless network [15]. However, the EH system dedicates some of the channel states (i.e. when $\left.\sum_{k=1}^{K} N_{0} \eta_{k} x_{k} \mu_{k}>\lambda\right)$ for broadcasting energy to the EHUs over the downlink.

It should be noticed that in this case, the achievable rate of EHU $k$ is determined by $\mathrm{E}\left[\tau_{k} \log _{2}\left(1+x_{k} P_{d, k}\right)\right]$, which can be achieved by using multiplexed multiple codebook scheme [14]. Specifically, an EHU in the fading state $\hat{\mathbf{x}}$ can achieve the rate $\tau_{k} \log _{2}\left(1+x_{k} P_{d, k}\right)$ by using a codeword from a state-specific codebook and a state-specific transmit power. Although single codebook with dynamic power allocation is still applicable to the perfect CSI case, the multiplexed multiple codebook scheme introduces much lower decoding delay (equal to the epoch duration $\mathrm{T}$ ).

\section{NUMERICAL RESULTS}

In this section we assume a WPCN operating in a block channel model with Rayleigh fading. The deterministic path loss is modeled using a reference path loss of $30 \mathrm{~dB}$ at reference distance of $1 \mathrm{~m}$, and path-loss exponent of $\alpha=3$, i.e.

$$
E\left[x_{k}(i) N_{0}\right]=10^{-3} D_{k}^{-3},
$$

where we used distance $D_{k}=12.6 \mathrm{~m}$. We assumed AWGN with power equal to $N_{0}=10^{-12} \mathrm{~W}$ [4] and use $P_{\max }=5 P_{a v g}$ where $P_{a v g}=5 \mathrm{~W}$ and $\eta_{k}=1$. Also, we assumed $N=3000$ symbols in each TDMA frame.

We obtain $\lambda$ and $\mu_{k}$ (needed in (8) - (10)) using an offline algorithm based on the ellipsoid method [16]. Practical online algorithms for calcu- lating $\lambda$ and $\mu_{k}$ can be easily obtained following the discussion from [4].

Figure 2 depicts the dependence of the achievable sum rate on the power allocated to the training phase $P_{p}$ and the number of training symbols $N_{p}$. As a benchmark we used the solutions (11) - (13) (marked as "Perfect CSI"). In Figure 2(a) the circuitry is assumed ideal, and thus $p_{c}=0$. Figure 2(a) shows that with proper choice of $P_{p}$, similar sum rate is achieved for a broad range of $N_{p}$ values. As in the conventional (non-EH) systems [6], the highest lower bound on the achievable sum rate is obtained for $N_{p}=1$ with small improvement compared to the case when to other $N_{p}$ are used. Lower value of $N_{p}$ requires higher value of $P_{p}$ to achieve high sum rates, but the increase in $P_{p}$ beyond the optimal value leads to substantial decrease in the sum rate. The combinations of $P_{p}$ and $N_{p}$ that achieve high sum rates, lead to small values of $\sigma_{k}^{2}$ such that $\Omega_{k} / \sigma_{k}^{2} \approx 200$.

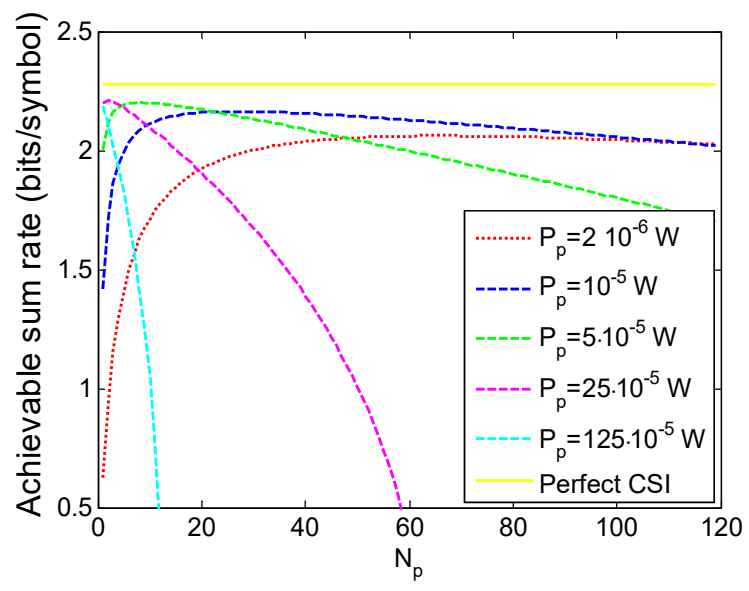

(a) $p_{c}=0$

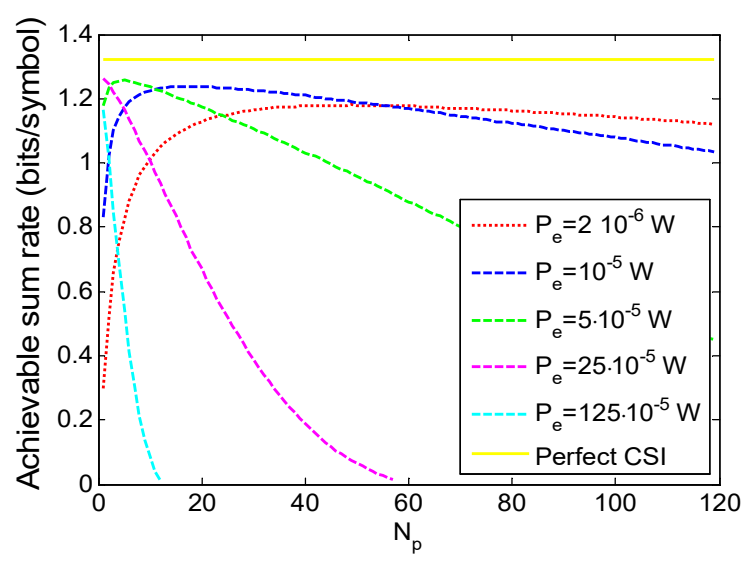

(b) $p_{c}=10^{-5}$

Fig. 2. Achievable sum rate vs. $N_{p}$ for $K=2$ 
The effect of non-ideal circuit power consumtion can be observed by comparing Figure 2(a) and Figure 2(b). A comparison of the achievable rates shows that the non-ideal circuit power consumption significantly reduces the maximum achievable rate. Namelly a part of the harvested energy istead for communication purposes, must be used for non-ideal circuit power consumption. Thus, the energy budget for communication is reduced. Additional observation is that for any value of $P_{p}$ in Figure 2(b) the value of $N_{p}$ which achieves the maximum achievable rate moves towards the lower number of pilot symbols compared to Figure 2(a). Furthermore, the achievable rates for values of $N_{p}$ higher than the $N_{p}$ which achieve the maximum achievable rate, have steeper decrease as $p_{c}$ increases. This is due to the fact that even in the training phase some energy is consumed in the non-ideal circuit power consumtion, and reducing the number of pilot symbols can reduce this nonproductive energy consumption.

Figure 3 shows the dependence of the achievable sum rate on the number of users $K$ when $p_{c}=$ 0 . It can be seen that as $K$ rises, the sum rate degradation due to the imperfect CSI increases, and the relative difference between the sum rates increases from $3.2 \%$ for $K=2$ to $4.3 \%$ for $K=5$. Part of this increase is a consequence of the decrease in the collected energy in each EHU (namely, by increasing $K$ the average power channel gain where an EHU receives energy is decreased towards $\Omega_{k}$ ), while keeping constant the energy required in the training phase (for Figure 3 the percentage of the energy used in the training phase of the total harvested energy ranges from $2.6 \%$ for $K=2$ to $3.18 \%$ for $K=5$ ). Additional sum rate difference is created by a decrease in $\tau_{g}$ (for Figure 3, $\tau_{g}$ decreases $0.8 \%$ for $K=5$ compared to $K=2$ ) and a reduced number of transmit opportunities (for Figure 3), BS occupies additional $0.2 \%$ of the epochs to transmit the same energy level for $K=5$ compared to $K=2$ ). The difference due to the reduction of $\tau_{g}$ and the reduction of the number of transmit opportunities can be alleviated to some extend by decreasing $N_{p}$.

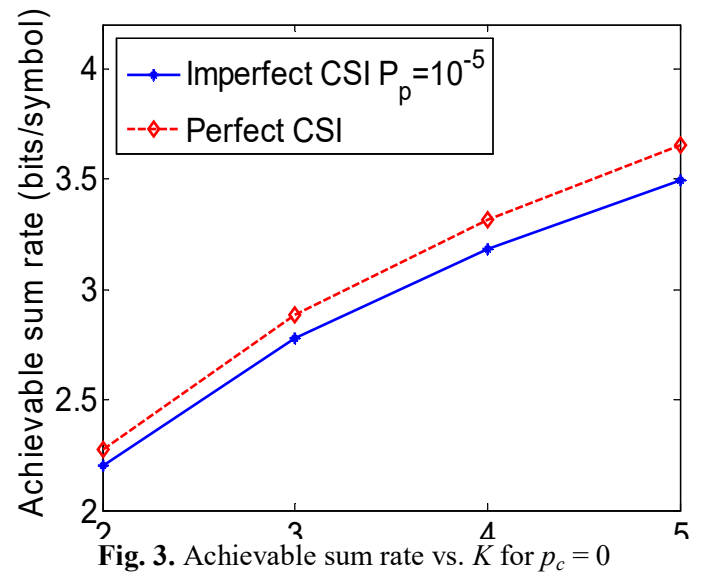

\section{CONCLUSION}

In this paper, we proposed a mechanism to maximize the achievable sum rate of WPCNs with imperfect CSI and non-ideal circiut power consumption. Numerical results indicate that the increased number of EHUs in the system leads to increase in the difference between the sum rates when perfect CSI is available and when imperfect CSI is available at both BS and the EHUs. To decrease this difference, small number of pilot symbols should be used and their power must be optimized according to this number. The non-ideal circiut power consumption significantly reduces the obtained sum rates and has the efect of additionally favoring the setings with lower number of pilot symbols for any pilot symbol power.

\section{REFERENCES}

[1] Gunduz, D., Stamatiou, K., Michelusi, N., Zorzi, M.: Designing intelligent energy harvesting communication systems, IEEE Communications Magazine, vol. 52, no. 1, pp. 210-216 (Jan. 2014).

[2] Ju, H., Zhang, R.: Throughput maximization in wireless powered communication networks, IEEE Transactions on Wireless Communications, vol. 13, no. 1, pp. 418-428 (Jan. 2014).

[3] Orhan, O., Gunduz, D., Erkip, E.: Throughput maximization for an energy harvesting communication system with processing cost, Proceedings of IEEE Information Theory Workshop 2012, Lausanne, Switzerland, pp. 8488.

[4] Hadzi-Velkov, Z., Nikoloska, I., Chingoska, H., Zlatanov, N.: Proportional fair scheduling in wireless networks with $\mathrm{RF}$ energy harvesting and processing cost, IEEE Communications Letters, vol. 20, no. 10, pp. 2107-2110 (Oct. 2016). 
[5] Pejoski, S., Hadzi-Velkov, Z., Schober, R.: Optimal power and time allocation for WPCNs with piece-wise linear EH model, IEEE Wireless Communications Letters, vol. 7, no. 3, pp. 364-367 (June 2018).

[6] Hassibi, B., Hochwald, B. M.: How much training is needed in multiple-antenna wireless links?, IEEE Transactions on Information Theory, vol. 49, no. 4, pp. 951963 (April 2003).

[7] Medard, M.: The effect upon channel capacity in wireless communications of perfect and imperfect knowledge of the channel, IEEE Transactions on Information Theory, vol. 46, no. 3, pp. 933-946 (May 2000).

[8] Klein, T. E., Gallager, R. G.: Power control for the additive white Gaussian noise channel under channel estimation errors, Proceedings of IEEE International Symposium on Information Theory, Washington, DC, 2001, pp. 304-304.

[9] Pejoski, S., Kafedziski, V.: Asymptotic capacity lower bound for an OFDM system with lasso compressed sensing channel estimation for bernoulli-gaussian channel, IEEE Communications Letters, vol. 19, no. 3, pp. 379382 (March 2015).

[10] Taesang Yoo, Goldsmith, A.: Capacity and power allocation for fading MIMO channels with channel estimation error, IEEE Transactions on Information Theory, vol. 52, no. 5, pp. 2203-2214 (May 2006).

[11] Lee, K., Hong, J. P.: Energy-efficient resource allocation for simultaneous information and energy transfer with imperfect channel estimation, IEEE Transactions on Vehicular Technology, vol. 65, no. 4, pp. 2775-2780 (April 2016).

[12] Zlatanov, N., Schober, R., Hadzi-Velkov, Z.: Asymptotically optimal power allocation for energy harvesting communication networks, IEEE Transactions on Vehicular Technology, vol. 66, no. 8, pp. 7286-7301 (2017).

[13] Caire, G., Shamai, S.: On the capacity of some channels with channel state information, IEEE Transactions on Inormation Theory, vol. 45, no. 6, pp. 2007-2019 (Sep. 1999).

[14] Goldsmith, A. J., Varaiya, P. P.: Capacity of fading channels with channel side information, IEEE Transactions on Information Theory, vol. 43, no. 6, pp. 1986-1992, Nov 1997.

[15] Knopp, R., Humblet, P. A.: Information capacity and power control in single-cell multiuser communications, Proceedings of IEEE International Conference on Communications, Seattle, 1995, pp. 331-335.

[16] Yu, W., Lui, R.: Dual methods for nonconvex spectrum optimization of multicarrier systems, IEEE Transactions on Communications, vol. 54, no. 7, pp. 1310-1322 (Jul. 2006).

[17] Rao, S.: Engineering optimization: Theory and Practice, Fourth Edition, Hoboken, New Jersey, John Wiley\& Sons, 2009.

\section{APPENDIX A}

\section{PROOF OF THEOREM 1}

To avoid nonconvexity from multiplication of variables, we modify (7) by introducing the auxiliary variables $a_{k}=P_{d, k} \tau_{k}$, and $e=P_{0} \tau_{0}$. In this case, the summ and of the objective function attains the form $f(x, y)=x \log \left(1+\frac{y}{x} \frac{1}{1+a y / x}\right)$ which is easily shown to be concave on the range of interest $(a, x, y \geq 0)$, by considering its Hessian. The constraints of (7) are all linear in $e, a_{k}, \tau_{0}$ and $\tau_{k}$. Thus, (7) is a convex optimization problem, and we can apply the Lagrange duality method to solve (7).

Using the calculus of variations [17, ch. 12], the functional for the modified version of problem (7) is given by (14), where $p(\hat{\mathbf{x}})$ is the probability density function of $\hat{\mathbf{x}}, \alpha(\hat{\mathbf{x}}), \beta(\hat{\mathbf{x}}), \delta_{k}(\hat{\mathbf{x}}), \gamma_{20}(\hat{\mathbf{x}})$, $\gamma_{10}(\hat{\mathbf{x}}), \quad v_{2 k}(\hat{\mathbf{x}}), \quad v_{1 k}(\hat{\mathbf{x}})$ and $\varepsilon(\hat{\mathbf{x}})$ are non-negative Lagrange multipliers associated to the constraints $C 3$ to $C 7$. They satisfy the following slackness conditions:

$$
\begin{aligned}
& \alpha(\hat{\mathbf{x}}) e=\beta(\hat{\mathbf{x}})\left(P_{\max } \tau_{0}-e\right)=0 \forall \hat{\mathbf{x}}, \\
& \delta_{j}(\hat{\mathbf{x}}) a_{k}=0 \quad \forall k, \hat{\mathbf{x}}, \\
& \gamma_{20}(\hat{\mathbf{x}}) \tau_{0}=\gamma_{10}(\hat{\mathbf{x}})\left(\tau_{g}-\tau_{0}\right)=0 . \\
& \forall \hat{\mathbf{x}}, v_{20}(\hat{\mathbf{x}}) \tau_{k}=v_{10}(\hat{\mathbf{x}})\left(\tau_{g}-\tau_{k}\right)=0, \\
& \forall k, \hat{\mathbf{x}},
\end{aligned}
$$

and,

$$
\varepsilon(\hat{\mathbf{x}})\left(\tau_{g}-\tau_{0}-\sum_{k=1}^{K} \tau_{k}\right)=0 \forall \hat{\mathbf{x}}
$$




$$
\begin{aligned}
A & =\int_{\hat{\mathbf{x}}}\left[\left(\sum_{k=1}^{K} \tau_{k} \log \left(1+\frac{\hat{x}_{k} \frac{a_{k}}{\tau_{k}}}{1+\sigma_{k}^{2} \frac{a_{k}}{\tau_{k}}}\right)+\sum_{k=1}^{K} \mu_{k}\left(N_{0} \eta_{k} z_{k} e-a_{k}-\left(P_{p}+p_{c}\right) \tau_{p}-\tau_{k} p_{c}\right)\right] p(\hat{\mathbf{x}})+\varepsilon(\hat{\mathbf{x}})\left(\tau_{g}-\tau_{0}-\sum_{k=1}^{K} \tau_{k}\right)\right. \\
& +\lambda\left(e-P_{a v g}\right) p(\hat{\mathbf{x}})-\beta(\hat{\mathbf{x}})\left(e-P_{\max } \tau_{0}\right)+\alpha(\hat{\mathbf{x}}) e+\sum_{k=1}^{K} \delta_{k}(\hat{\mathbf{x}}) a_{k}+\gamma_{20}(\hat{\mathbf{x}}) \tau_{0}-\gamma_{10}(\hat{\mathbf{x}})\left(\tau_{0}-\tau_{g}\right)+\sum_{k=1}^{K} v_{2 k}(\hat{\mathbf{x}}) \tau_{k} \\
& \left.-\sum_{k=1}^{K} v_{1 k}(\hat{\mathbf{x}})\left(\tau_{k}-\tau_{g}\right)\right] d \hat{\mathbf{x}}=\int_{\hat{\mathbf{x}}} \mathrm{L} d \hat{\mathbf{x}} .
\end{aligned}
$$

To maximize the functional in (14) we have to set the first derivatives of $\mathrm{L}$ (the function under the integral in (14)) with respect to $e, \tau_{0}, a_{k}$, and $\tau_{k}$ to zero. By doing so, and substituting

$$
b(\hat{\mathbf{x}})=1+\hat{x}_{k} \frac{a_{k}}{\tau_{k}} /\left(1+\frac{\sigma_{k}^{2} a_{k}}{\tau_{k}}\right),
$$

we obtain:

$$
\frac{d \mathrm{~L}}{d e}=\left(\sum_{k=1}^{K} \mu_{k} N_{0} \eta_{k} z_{k}-\lambda\right) p(\hat{\mathbf{x}})-\beta(\hat{\mathbf{x}})+\alpha(\hat{\mathbf{x}})=0
$$

$$
\frac{d \mathrm{~L}}{d \tau_{0}}=-\varepsilon(\hat{\mathbf{x}})+\beta(\hat{\mathbf{x}}) P_{\max }+\gamma_{20}(\hat{\mathbf{x}})-\gamma_{10}(\hat{\mathbf{x}})=0
$$

$$
\frac{d \mathrm{~L}}{d a_{k}}=\left(\frac{\hat{x}_{k}}{\left(1+\sigma_{k}^{2} \frac{a_{k}}{\tau_{k}}\right)^{2}} \frac{1}{b(\hat{\mathbf{x}})}-\mu_{k}\right) p(\hat{\mathbf{x}})+\delta_{k}(\hat{\mathbf{x}})=0
$$

$$
\begin{aligned}
\frac{d \mathrm{~L}}{d \tau_{k}}= & \left(\log (b(\hat{\mathbf{x}}))-\frac{a_{k}}{\tau_{k}} \frac{\hat{x}_{k} /\left(1+\sigma_{k}^{2} \frac{a_{k}}{\tau_{k}}\right)^{2}}{b(\hat{\mathbf{x}})}-p_{c} \mu_{k}\right) p(\hat{\mathbf{x}})- \\
& -\varepsilon(\hat{\mathbf{x}})+v_{2 k}(\hat{\mathbf{x}})-v_{1 k}(\hat{\mathbf{x}})=0
\end{aligned}
$$

$$
\begin{aligned}
& \text { From (15), when } \sum_{k=1}^{K} \mu_{k} N_{0} \eta_{k} z_{k}>\lambda \text { we have } \\
& \beta(\hat{\mathbf{x}})>0 \text {, i.e. } P_{0}=P_{\max } \text { and when }
\end{aligned}
$$

$\sum_{k=1}^{K} \mu_{k} N_{0} \eta_{k} z_{k}<\lambda$ we have $\alpha(\hat{\mathbf{x}})>0$ i.e. $P_{0}=0$, leading to (8).

When $P_{d, k}>0$, i.e. $\delta_{k}(\hat{\mathbf{x}})=0$, from (17) we calculate $a_{k} / \tau_{k}$ as a function of $\mu_{k}, \sigma_{k}^{2}$ and $\hat{x}_{k}$ i.e. $a_{k} / \tau_{k}=f_{\mu_{k}, \sigma_{k}^{2}}\left(\hat{x}_{k}\right)$. When a single epoch is shared by an EH and an IT phase, (16) must be satisfied with $\gamma_{20}(\hat{\mathbf{x}})=\gamma_{10}(\hat{\mathbf{x}})=0$, so we obtain $\varepsilon(\hat{\mathbf{x}})=\beta(\hat{\mathbf{x}}) P_{\max }$. At the same time, at least for a single $k$ eq. (18) must be satisfied with $v_{2 k}(\hat{\mathbf{x}})=v_{1 k}(\hat{\mathbf{x}})=0$. The probability of occurrence of this case is zero, because $\hat{x}_{k}$ follow continuous distributions. Thus, the whole epoch is allocated either to an $\mathrm{EH}$ or to an IT phase.

When the epoch is used for IT phase, and more than a single EHU is transmitting, then eq. (18), with $v_{2 k}(\hat{\mathbf{x}})=v_{1 k}(\hat{\mathbf{x}})=0$, must be satisfied for more than one $k$, which happens with probability zero due to the continuous nature of $\hat{x}_{k}$. Thus a single EHU uses the epoch. The power of the active EHU is obtained as $P_{d, k}=f_{\mu_{k}, \sigma_{k}^{2}}\left(\hat{x}_{k}\right)$, where $f_{\mu_{k}, \sigma_{k}^{2}}\left(\hat{x}_{k}\right)$ is obtained by solving (17), and has the form of (9), and, using the derivation from [4] and eq. (18), the EHU with maximum:

$$
g_{k}=\log (b(\hat{\mathbf{x}}))-\frac{a_{k}}{\tau_{k}} \frac{\hat{x}_{k} /\left(1+\sigma_{k}^{2} \frac{a_{k}}{\tau_{k}}\right)^{2}}{b(\hat{\mathbf{x}})}-p_{c} \mu_{k},
$$

is active. Substituting $\mu_{k}$ calculated from (17) for $\delta_{k}(\hat{\mathbf{x}})=0$ (i.e., $P_{d, k}>0$ ), and using the definition of $a_{k}$ in eq. (19), we obtain (10) which concludes the proof. 